\title{
INTEGRAL OPERATORS AND THE COMPACTNESS OF INDUCED REPRESENTATIONS
}

\author{
BY \\ ROBERT C. BUSBY, IRWIN SCHOCHETMAN AND HARVEY A. SMITH
}

\begin{abstract}
Integral operators are investigated which are compositions of multiplication by bounded vector valued functions and convolutions with vector valued functions. All of the functions are based on a fixed locally compact group. Conditions are given under which certain of these operators are compact. As an application of these conditions we consider induced representations of twisted group algebras (these are generalizations of representations of groups induced from closed normal subgroups in the sense of Mackey and include these as special cases) and we give necessary and sufficient conditions for these representations to be compact (that is, to consist entirely of compact operators).
\end{abstract}

1. Introduction. One of the more important concepts in the theory of unitary representations of locally compact groups is that of induced representation. Another is that of a compact (or C.C.R) representation-one which when lifted to the group algebra yields only compact operators. In this paper we will prove some general theorems which will in particular yield necessary and sufficient conditions for the representation induced from a closed normal subgroup of a second countable locally compact group to be compact.

In $\$ \S 2$ and 3, we investigate a class of integral operators with operator-valued kernels acting on vector-valued functions. (The simplest instance of the operators we consider is an operator $K$ on $L^{2}(G)$ defined by $K(g)=h \cdot(f * g)$ where $f$ in $L^{1}(G)$ acts on $L^{2}(G)$ by convolution and $h$ in $L^{\infty}(G)$ acts by pointwise multiplication. Our results, in particular, yield necessary and sufficient conditions on $h$ for every operator of this form to be compact.)

In $\S 4$ we review the concept of twisted group algebra as developed in [4]. This general type of Banach *-algebra is constructed from an action of a locally compact second countable group on a Banach *-algebra. Included as special cases are the group algebra of a group extension (considered in terms of the quotient group acting on the group algebra of the subgroup), algebras associated with covariant representations of $C^{*}$-algebras and locally compact automorphism groups, and other algebras previously constructed in the literature using the action of a locally compact transformation group. This general type of algebra provides a natural

Received by the editors May 23, 1970.

AMS 1969 subject classifications. Primary 4650, 2260; Secondary 4256, 4680.

Key words and phrases: Compact integral operator, C.C.R. representation, induced representation, twisted group algebra, covariance algebra.

Copyright (C) 1972, American Mathematical Society 
setting for the concept of induced representation. We examine such representations and show that the results of $\$ 3$ apply to give necessary and sufficient conditions for induced representations to be compact.

Finally, in $\$ 5$ we specialize the conditions to give the promised results for induced representations of groups. We then augment these results with some weak containment arguments and give strengthened theorems under additional hypotheses (certain normal subgroups are type I and regularly embedded).

2. General remarks on integral operators. Let $(X, S, m)$ be a measure space where either $S$ is a sigma ring of subsets of the set $X$ and $m$ is sigma-finite on $S$, or $X$ is a locally compact Hausdorff space, $S$ is the sigma ring of Borel sets, and $m$ is a positive Radon measure. Let $(Y, R, n)$ be the product measure space of $(X, S, m)$ with itself, $E$ be a Banach space, $C(E)$ the compact operators on $E$, and let $A(X)$ be either the complex linear space generated by the characteristic functions of sets in $S$ of finite $m$-measure or the space of continuous complex-valued functions with compact support on $X$, depending on the interpretation of $(X, S, m)$. Finally, let $L^{2}(X, S, m, E)$ and $L^{2}(Y, R, n, C(E))$ be the appropriate spaces of Bochner integrable vector-valued functions $\left({ }^{1}\right)$. Then the following facts are either well known or are simple generalizations of well-known results. As a general reference see [6]:

(1) If $k(x, y)$ is in $L^{2}(Y, R, n, C(E))$, the operator $K$ on $L^{2}(X, S, m, E)$ given by

$$
(K f)(x)=\int k(x, y) f(y) d m(y)
$$

is a bounded linear operator and

$$
\|K\|^{2} \leqq \int\|k(x, y)\|^{2} d n(x, y)
$$

(2) The linear span in $L^{2}(Y, R, n, C(E))$ of elements of the form $k(x, y)$ $=h(x) g(y) B ; h$ and $g$ in $A(X), B$ in $C(E)$; is a dense set.

Proposition 2.1. The operator $K$ defined above is compact.

Proof. $K$ exists by remark (1), and by remark (2) we may assume that $k(x, y)$ $=h(x) g(y) B ; h, g$ in $A(X)$, and $B$ in $C(E)$. Then for $f$ in $L^{2}(X, S, m, E)$,

$$
[K f](x)=h(x) B\left[\int g(y) f(y) d m(y)\right]=h(x) B v_{f}
$$

where $v_{f}$ is in $E$. Now let $\left\{f_{j}\right\}$ be a bounded sequence in $L^{2}(X, S, m, E)$ and $v_{j}$ be the corresponding $v_{f}$ 's. It is easily seen that $\left\|v_{j}\right\| \leqq C\left\|f_{j}\right\|_{2}$ so that $\left\{v_{j}\right\}$ is a bounded sequence in $E$ and thus some subsequence $\left\{B v_{i}\right\}$ of $\left\{B v_{j}\right\}$ converges to $v$ in $E$. If $f(x)=h(x) v$, then $f$ is in $L^{2}(X, S, m, E)$ and $\left\|K f_{j}-f\right\|_{2}<C_{1}\left\|B v_{j}-v\right\|$, which completes the proof.

( ${ }^{1}$ In the first instance we mean the norm integrable functions which are measurable with respect to the norm Borel structure of $E$. In the second instance we take the functions to be strongly measurable. 
We remark now (without proof) that one can also show that if $E$ is a Hilbert space, HS $(E)$ the Hilbert-Schmidt operators on $E, k$ is in $L^{2}(Y, R, n, \mathrm{HS}(E))$, and $K$ is defined as before, then $K$ is a Hilbert-Schmidt operator on $L^{2}(X, S, m, E)$ and the Hilbert-Schmidt norm of $K$ is the $L^{2}$-norm of $k$.

Now suppose that $G$ is a locally compact group, $m$ is (a left) Haar measure on $G$, and $\Delta$ is the corresponding modular function. Let $H$ be a Hilbert space and $k: G \times G \rightarrow C(H)$ have the following properties:

(1) $k$ is norm bounded and Borel measurable.

(2) There exist compact sets $E_{1}$ and $E_{2}$ in $G$ such that $k$ vanishes outside of $E_{1} \times E_{2}$.

Proposition 2.2. The operator $K$ on $L^{2}(G, m, H)$ given by

is compact.

$$
[K f](x)=\int_{G} k(x, y) f(x y) d m(y)
$$

Proof. Clearly

$$
\begin{aligned}
{[K f](x) } & =\int_{G} k\left(x, x^{-1} y\right) f(y) d m(y) \\
& =\int_{G} k^{\prime}(x, y) f(y) d m(y) .
\end{aligned}
$$

Since $k^{\prime}$ is norm bounded and vanishes outside the compact set $E_{1} \times E_{1} E_{2}, k^{\prime}$ is in $L^{2}(G, m, C(H))$ and previous results yield the conclusion.

Now let $K$ be defined as above where this time $k: G \times G \rightarrow C(H)$ satisfies

(1) $k$ is norm bounded and Borel measurable.

(2) There is a compact set $E$ such that if $y$ is not in $E, k(x, y)=0$.

(3) $\|k(x, y)\|$ tends to zero uniformly in $y$ as $x$ goes to infinity.

THEOREM 2.3. $K$ is compact.

Proof. Let $E_{n}$ be the set of all $x$ in $G$ such that $\|k(x, y)\| \leqq 1 / n$ for all $y$ in $G$, let $k_{n}=k$ on $E_{n} \times E$ and vanish elsewhere, and let $K_{n}$ be the operator formed from $k_{n}$ as $K$ is from $k$. Since $K-K_{n}$ corresponds to $k-k_{n}$, which is bounded with compact support, Proposition 1.2 implies that $K-K_{n}$ is compact. We need only show that $\left\|K_{n}\right\|$ tends to zero. Let $f$ be in $L^{2}(G, m, H)$. Then

$$
\begin{aligned}
\left\|K_{n} f\right\|^{2} & =\int_{G}\left\|K_{n} f(x)\right\|^{2} d m(x) \\
& \leqq \int_{G}\left(\int_{G}\left\|k_{n}(x, y)\right\|\|f(x y)\| d m(y)\right)^{2} d m(x) \\
& \leqq \int_{G}\left(\int_{E}\left\|k_{n}(x, y)\right\|^{2} d m(y)\right)\left(\int_{E}\|f(x y)\|^{2} d m(y)\right) d m(x) \\
& \leqq\left\|k_{n}\right\|_{\infty}^{2} m(E) \int_{E} \int_{G}\|f(x y)\|^{2} d m(x) d m(y) \\
& \leqq\left\|k_{n}\right\|_{\infty}^{2} m(E)^{2} s\|f\|^{2} \leqq\left(c^{2} / n^{2}\right)\|f\|^{2}
\end{aligned}
$$


(where $s$ is the supremum of $\Delta(y)^{-1}$ on the compact set $E$ ). Thus $\left\|K_{n}\right\| \leqq c / n$ which tends to zero. This completes the proof. A special case of the above operator was investigated in [1].

3. Operators of product-convolution type. Let $R$ be the real line with Lebesgue measure, and let $L^{1}(R)$ and $L^{\infty}(R)$ act on $L^{2}(R)$ by convolution and multiplication respectively. It is not hard to show that in the first case we never get compact operators and in the second case we rarely do-never for (nonzero) continuous functions. It frequently happens however that operators arise which are compositions of the two above mentioned types, and one may ask when these are compact. In a much more general context, we will give necessary and sufficient conditions on a member of $L^{\infty}(R)$ so that composing it as an operator with the operator defined by any function in $L^{1}(R)$ will always yield a compact operator.

Let $C(H), B(H)$ and $U(H)$ be the compact, bounded, and unitary operators respectively on a Hilbert space $H$, let $G$ be a locally compact second countable group, let $m$ and $\Delta$ be as in $\S 2$, let $h, f, g$ be in $L^{\infty}(G, m, C(H)), L^{1}(G, m, B(H))$, and $L^{2}(G, m, H)$ respectively. If $A$ is a Borel measurable function from $G \times G$ to $U(H)$, we can define operators $Q_{h}$ (multiplication) and $K_{A f}$ (generalized weighted right convolution) on $L^{2}(G, m, H)$ by

and

$$
\left(Q_{h} g\right)(x)=h(x) g(x)
$$

$$
\left(K_{A f} g\right)(x)=\int_{G} A(x, y) f(y) g(x y) \Delta(y)^{1 / 2} d m(y) .
$$

Proposition 3.1. $Q_{h}$ and $K_{A f}$ are bounded linear operators. Furthermore, $\left\|Q_{h}\right\|$ $=\|h\|_{\infty}$ and $\left\|K_{A f}\right\| \leqq\|f\|_{1}$.

Proof. The assertions concerning $Q_{h}$ and the linearity of $K_{A f}$ are trivial.

$$
\begin{aligned}
\left\|K_{A f} g\right\|^{2} & \leqq \int\left[\int\|f(y)\|\|g(x y)\| \Delta(y)^{1 / 2} d m(y)\right]^{2} d m(x) \\
& =\int\left[\left(\int\|f(y)\|\|g(x y)\| \Delta(y)^{1 / 2} d m(y)\right)\right. \\
\left.\cdot\left(\int\|f(z)\|\|g(x z)\| \Delta(z)^{1 / 2} d m(z)\right)\right] d m(x) & \\
& \leqq \iint\left[\left(\int\|g(x y)\|\|g(x z)\| d m(x)\right)\|f(y)\|\|f(z)\| \Delta(y z)^{1 / 2}\right] d m(y) d m(z) \\
& \leqq \iint\left[\left(\int\|g(x y)\|^{2} d m(x)\right)^{1 / 2}\left(\int\|g(x z)\|^{2} d m(x)\right)^{1 / 2}\right. \\
& \leqq\|g\|^{2}\|f\|_{1}^{2} .
\end{aligned}
$$

Thus $\left\|K_{A f}\right\| \leqq\|f\|_{1}$. 
Definition 3.2. With notation as above, an operator on $L^{2}(G, m, H)$ of the form $Q_{h} K_{A f}$ is said to be of product-convolution type.

Our goal is to give conditions in terms of $h$ so that for $A$ fixed, $Q_{h} K_{A f}$ is compact for all $f$ in $L^{1}(G, m, B(H))$.

Definition 3.3. A (Borel) subset $E$ of a locally compact second countable group $G$ will be called thin at infinity if for any compact neighborhood $I$ of the identity in $G$, the function $t_{I}(x)=m(I x \cap E) / \Delta(x)$ vanishes at infinity.

If $G$ is unimodular, this condition just means that the relative measure of $E$ intersected with $x$-translates of $I$ goes to zero as $x$ goes to infinity. An example of a set thin at infinity in the real line is $E=\bigcup_{n}\left(a_{n}, a_{n}+b_{n}\right)$ where $a_{n}$ and $b_{n}$ are positive, $a_{n}$ tends to infinity, and $b_{n}$ tends to zero as $n$ tends to infinity.

Definition 3.4. Let $B$ be a Banach space and let $T$ be in $L^{\infty}(G, m, B)$. For all $c>0$ let $E(T, c)$ be the set of all $x$ in $G$ such that $\|T(x)\| \geqq c$. We say that $T$ almost vanishes at infinity if $E(T, c)$ is thin at infinity for all $c>0$.

THEOREM 3.5. Let $G$ be a second countable group and let $h$ be in $L^{\infty}(G, m, C(H))$ for a Hilbert space $H$. If $h^{\sim}(x)=h\left(x^{-1}\right)$ almost vanishes at infinity, then the operator $K=Q_{h} K_{A f}$ is compact for any $f$ in $L^{1}(G, m, B(H))$ and $A$ in $L^{\infty}(G \times G, m \times m, U(H))$.

Proof. Let $r_{c}$ be the characteristic function of $E\left(h^{\sim}, c\right)$. Notice that $\left\|h^{\sim}-r_{c} h^{\sim}\right\|_{\infty}$ converges to zero as $c$ tends to zero. We may therefore assume that the support of $h$ is contained in $E(h, c)$ for some $c>0$. Similarly, by density arguments, we may assume that $f(x)=T F(x)$ where $T$ is in $B(H)$ and $F$ is a positive, real valued, continuous function on $G$ with compact support $I$ with $\|F\|_{\infty} \leqq 1$. Then

$$
\begin{aligned}
(K g)(x) & =\left(Q_{h} K_{A f} g\right)(x) \\
& =\int_{G} h(x) A(x, y) f(y) g(x y) \Delta(y)^{1 / 2} d m(y) \\
& =\int_{G} h(x) A\left(x, x^{-1} y\right) f\left(x^{-1} y\right) \Delta(x)^{-1 / 2} \Delta(y)^{1 / 2} g(y) d m(y) \\
& =\int_{G} k(x, y) g(y) d m(y) .
\end{aligned}
$$

Let

$$
\begin{aligned}
k(x, y) & =h(x) A\left(x, x^{-1} y\right) T F\left(x^{-1} y\right) \Delta(x)^{-1 / 2} \Delta(y)^{1 / 2} \\
& =C(x, y) F\left(x^{-1} y\right) \Delta(x)^{-1 / 2} \Delta(y)^{1 / 2} .
\end{aligned}
$$

Then $C(x, y)$ is in $C(H)$, and $\|C(x, y)\| \leqq\|h(x)\|\|T\|$. We now look at the operator $K^{*} K$. The function

$$
\begin{aligned}
p(x, y) & =\int k(z, x)^{*} k(z, y) d m(z) \\
& =\int\left[C(z, x)^{*} C(z, y) F\left(z^{-1} x\right) F\left(z^{-1} y\right) \Delta(x)^{1 / 2} \Delta(y)^{1 / 2} \Delta(z)^{-1}\right] d m(z)
\end{aligned}
$$


exists since the integrand in question is bounded and has compact support. It is then not difficult to show that if $g$ is in $L^{2}(G, m, H), \int_{G} p(x, y) g(y) d m(y)$ exists and defines a bounded linear operator. Direct computation shows that this operator is $K^{*} K$. We conclude that

$$
\begin{aligned}
\left(K^{*} K g\right)(x) & =\int_{G} p(x, x y) g(x y) d m(y) \\
& =\int_{G} r(x, y) g(x y) d m(y)
\end{aligned}
$$

and

$$
\begin{aligned}
\|r(x, y)\| & \leqq\|T\|^{2} \Delta(x) \Delta(y)^{1 / 2} \int\left[\left\|h\left(z^{-1}\right)\right\|^{2} F(z x) F(z x y)\right] d m(z) \\
& \leqq\|T\|^{2}\|h\|_{\infty}^{2} \Delta(y)^{1 / 2} m\left(I x^{-1} \cap I y^{-1} x^{-1} \cap E\left(h^{\sim}, c\right)\right) / \Delta\left(x^{-1}\right) \\
& \leqq\|T\|^{2}\|h\|_{\infty}^{2} \Delta(y)^{1 / 2} m\left(\left(I \cap I y^{-1}\right) x^{-1} \cap E\left(h^{\sim}, c\right)\right) / \Delta\left(x^{-1}\right) .
\end{aligned}
$$

This computation shows that $r(x, y)=0$ if $y$ is not in $I^{-1} I$ (a compact set). Since $\Delta(y)^{1 / 2}$ is bounded on $I^{-1} I, r(x, y)$ is norm bounded. Finally, since $m\left(I^{-1} I x^{-1} \cap E\left(h^{\sim}, c\right)\right) / \Delta\left(x^{-1}\right)$ vanishes at infinity by hypothesis we see that $r(x, y)$ vanishes at infinity uniformly in $y$ as $x$ goes to infinity. Now Theorem 2.3 shows that $K^{*} K$ is a compact operator. Then by spectral theory, $\left(K^{*} K\right)^{1 / 2}$ is compact. Finally $K=U\left(K^{*} K\right)^{1 / 2}$ for some partial isometry $U$ by the polar decomposition of operators, and thus $K$ is compact. This completes the proof.

We will now proceed to prove a converse of Theorem 3.5 under slightly stronger hypotheses.

Definition 3.6. Suppose that $E \subset G$ is Borel, and $V$ is a compact, symmetric neighborhood of the identity $e$ in $G$. We say that $\left\{V x_{n}\right\}, 1 \leqq n<\infty$, is (for $E$ ) a fundamental essential sequence (F.E. sequence) of $V$ translates if:

(1) $x_{n} \rightarrow \infty$ in $G$,

(2) $\inf _{n}\left\{m\left(V x_{n} \cap E\right) / \Delta\left(x_{n}\right)\right\}>0$,

(3) for all $n>1, x_{n}$ is not in $\bigcup_{k<n} V^{6} x_{k}$,

(4) for all $n, x_{n}$ is not in $V^{3}$.

LEMmA 3.7. The following statements are equivalent for $E \subset G$ :

(1) $E$ is not thin at infinity.

(2) For every compact, symmetric neighborhood $V$ of the identity, there is (for E) an F.E. sequence of $V$ translates.

Proof. (2) $\Rightarrow(1)$ is immediate from the definitions. (1) $\Rightarrow(2)$. Negating the definition of thin at infinity, we see that there must be at least one compact neighborhood $W$ of $e$ and a positive number $d$ such that the following property holds: For every compact set $C$ there is an $x$ not in $C$ such that $m(W x \cap E) \geqq d \Delta(x)$.

If we choose any compact, symmetric neighborhood $V$ of $e$, we can find $y_{1}, \ldots, y_{s}$ in $W$ such that $W \subset \bigcup_{k} V y_{k}$. Let $a=\max _{k} \Delta\left(y_{k}\right)$. Choose a sequence $\left\{x_{n}\right\}$ such that, 
for all $n, s \cdot m\left(V y_{k} x_{n} \cap E\right) \geqq d \Delta\left(x_{n}\right), x_{n}$ is not in $\bigcup_{p<n}\left(y_{k}^{-1} V^{6} y_{k} x_{p}\right), x_{n}$ is not in $W^{-1} V^{3}$ and $x_{n} \rightarrow \infty$ in $G$, for some fixed $k$. Writing $z_{n}=y_{k} x_{n}$, we have $z_{n} \rightarrow \infty$, $z_{n} \notin V^{3}$ and

$$
m\left(V z_{n} \cap E\right) \geqq \frac{d \Delta\left(x_{n}\right)}{s} \geqq \frac{d \Delta\left(z_{n}\right)}{(a s)} .
$$

Thus (a) $\inf \left\{m\left(V z_{n} \cap E\right) / \Delta\left(z_{n}\right)\right\} \geqq d /(a s)>0$ and

(b) since $x_{n}$ is not in $\bigcup_{p<n} y_{k}^{-1} V^{6} y_{k} x_{p}, z_{n}$ is not in $\bigcup_{p<n} V^{6} z_{p}$ and since $V$ is arbitrary, (2) holds.

Now, continuing with previous notation, we consider the operator $K=Q_{h} K_{A f}$ on $L^{2}(G, m, H)$. We now discuss a joint property of $h$ and $A$, for the case when $h^{\sim}$ does not almost vanish at infinity, which is a piecewise uniformly small variation condition.

Suppose that $h^{\sim}$ does not almost vanish at infinity and $c>0$ is any constant such that the set $E_{c}$ of all $x$ with $\left\|h\left(x^{-1}\right)\right\| \geqq c$ is not thin at infinity. We know by Lemma 3.7 that there is for $E_{c}$ an F.E. sequence of translates of $V$ for every compact, symmetric neighborhood $V$ of the identity $e$.

Definition 3.8. Suppose that for each $E_{c}$ above there is a compact, symmetric neighborhood $V$ of $e$ and an F.E. sequence $\left\{V x_{n}\right\}$ for $E_{c}$ such that

$$
\left\{h\left(x^{-1}\right) A\left(x^{-1}, y\right): x \in V x_{n}, y \in V\right\}
$$

lies in a sphere of radius $c / 4$ in $C(H)$ for all $n$. Then we say that $h$ and $A$ are regulated.

Without much trouble one can show that $h$ and $A$ will be regulated if $h\left(x^{-1}\right) A\left(x^{-1}, y\right)$ is uniformly continuous on $U\left(V x_{n} \times V\right)$, in particular if $h\left(x^{-1}\right) A\left(x^{-1}, y\right)$ is uniformly continuous on $G \times G$. We now have the following converse of Theorem 3.5.

THEOREM 3.9. Let $G$ be a second countable locally compact group, $H$ a Hilbert space, $h$ a function in $L^{\infty}(G, m, C(H)), A$ in $L^{\infty}(G \times G, m \times m, U(H))$.

If $h^{\sim}$ does not almost vanish at infinity, and if $h$ and $A$ are regulated, then there is an $f$ in $L^{1}(G, m, B(H))$ such that $Q_{h} K_{A f}$ is not compact.

Proof. For some $c>0$ the set $E_{c}$ of all $x$ such that $\left\|h\left(x^{-1}\right)\right\| \geqq c$ is not thin at infinity. Since $h$ and $A$ are regulated we can choose a compact symmetric neighborhood $V$ of $e$ and a sequence $\left\{x_{n}\right\}$ such that

(1) $V x_{n}$ is an F.E. sequence for $E_{c}$.

(2) $h\left(x^{-1}\right) A\left(x^{-1}, y\right)$ varies by less than $c / 4$ on each $V x_{n} \times V$.

Let $\inf _{n}\left(m\left(V x_{n} \cap E_{c}\right) / \Delta\left(x_{n}\right)\right)=d>0$. Let $f=m(V)^{-1} C_{V} I$ where $I$ is the identity operator on $H$ and $C_{V}$ is the characteristic function of $V$. Choose, for each $n$, a point $y_{n}$ in $V x_{n}$ such that $\left\|h\left(y_{n}^{-1}\right) A\left(y_{n}^{-1}, e\right)\right\|=\left\|h\left(y_{n}^{-1}\right)\right\| \geqq c$. For each $n$, there is a normalized vector $v_{n}$ in $H$ such that $\left\|h\left(y_{n}^{-1}\right) A\left(y_{n}^{-1}, e\right) v_{n}\right\| \geqq 3 c / 4$.

For each $n$ let $g_{n}$ be defined by

$$
g_{n}(x)=v_{n} g\left(x^{-1} x_{n}^{-1}\right) \Delta\left(x_{n}\right)^{-1 / 2} \Delta(x)^{-1 / 2}
$$


where $g$ is the characteristic function of $V^{2}$. Then $g_{n}$ is in $L^{2}(G, m, H)$ for each $n$ and $f$ is in $L^{1}(G, m, B(H))$. Since

$$
\begin{aligned}
\left\|g_{n}\right\|_{2}^{2} & =\int_{G}\left\|g_{n}(x)\right\|^{2} d m(x) \\
& =\int\left|g\left(x^{-1} x_{n}^{-1}\right)\right|^{2} \Delta\left(x_{n}\right)^{-1} \Delta(x)^{-1} d m(x)=\|g\|_{2}^{2},
\end{aligned}
$$

the sequence $\left\{g_{n}\right\}$ is bounded. We will be done if we can show that the sequence $\left\{Q_{n} K_{A f} g_{n}\right\}$ has no converging subsequences. By definition of $f$ and $g_{n}$, the support of $Q_{n} K_{A f} g_{n}$ is contained in $x_{n}^{-1} V^{3}$. If $z$ is in $x_{n}^{-1} V^{3} \cap x_{m}^{-1} V^{3}(m<n)$, then $x_{n}$ is in $V^{6} x_{m}$ which violates our hypotheses. This means that the supports of the functions $Q_{h} K_{A f} g_{n}$ are disjoint. Finally we have

$$
\begin{aligned}
\left\|Q_{h} K_{A f} g_{n}\right\|_{2}^{2} & =\int\left\|\int h(x) A(x, y) f(y) g_{n}(x y) \Delta(y)^{1 / 2} d m(y)\right\|^{2} d m(x) \\
& =\int\left[\left\|\int h^{\sim}(x) A\left(x^{-1}, y\right) f(y) v_{n} g\left(y^{-1} x x_{n}^{-1}\right) d m(y)\right\|^{2}\right] d m(x) / \Delta\left(x_{n}\right) \\
& \geqq \int_{V x_{n} \cap E_{c}}\left\|h^{\sim}(x) \int_{V} m(V)^{-1} A\left(x^{-1}, y\right) v_{n} d m(y)\right\|^{2} d m(x) / \Delta\left(x_{n}\right)
\end{aligned}
$$

(since if $y$ is in $V$ and $x$ in $V x_{n}, y^{-1} x x_{n}^{-1}$ is in $V^{2}$ and so $m(V) f(y)=I$ and $\left.g\left(y^{-1} x x_{n}^{-1}\right)=1\right)$.

Let $w_{n}(x)=m(V)^{-1} \int_{V} h\left(x^{-1}\right) A\left(x^{-1}, y\right) v_{n} d m(y)$. Then

$$
\begin{aligned}
\| w_{n}(x)-h\left(y_{n}^{-1}\right) A( & \left.y_{n}^{-1}, e\right) v_{n} \| \\
& =m(V)^{-1}\left\|\int_{V}\left[h\left(x^{-1}\right) A\left(x^{-1}, y\right) v_{n}-h\left(y_{n}^{-1}\right) A\left(y_{n}^{-1}, e\right) v_{n}\right] d m(y)\right\| \\
& \leqq \sup _{y \in V}\left\|h\left(x^{-1}\right) A\left(x^{-1}, y\right)-h\left(y_{n}^{-1}\right) A\left(y_{n}^{-1}, e\right)\right\| \leqq c / 4
\end{aligned}
$$

by hypothesis for $x$ in $V x_{n}$.

Thus,

$$
\begin{aligned}
\left\|Q_{h} K_{A f} g_{n}\right\|_{2}^{2} & \geqq \int_{V x_{n} \cap E_{c}}\left\|w_{n}(x)\right\|^{2} d m(x) / \Delta\left(x_{n}\right) \\
& \geqq \int_{V x_{n} \cap E_{c}}\left(\left\|h\left(y_{n}^{-1}\right) A\left(y_{n}^{-1}, e\right) v_{n}\right\|-\frac{c^{2}}{4}\right) d m(x) / \Delta\left(x_{n}\right) \\
& \geqq \frac{c^{2}}{4} \frac{m\left(V x_{n} \cap E_{c}\right)}{\Delta\left(x_{n}\right)} \geqq \frac{d c^{2}}{4}>0 .
\end{aligned}
$$

The functions $Q_{n} K_{A f} g_{n}$ are uniformly bounded below in norm and have disjoint supports, so the sequence can have no converging subsequences and the proof is complete. 
We close this section by showing that in certain cases we can eliminate the extra hypothesis in Theorem 3.9. The following result includes in particular the case of the real line mentioned at the start of this section.

THEOREM 3.10. If $G$ is a locally compact, second countable group, $H$ is a finite dimensional Hilbert space, $h$ and $f$ are as previously indicated, and $Q_{h} K_{f}$ is defined on $L^{2}(G, m, H)$ by

$$
\left[Q_{h} K_{f} g\right](x)=\int_{G} h(x) f(y) g(x y) \Delta(y)^{1 / 2} d m(y),
$$

then $Q_{h} K_{f}$ is compact for all $f$ in $L^{1}(G, m, H)$ if and only if $h^{\sim}$ almost vanishes at infinity.

Proof. The "if" part follows from Theorem 3.5. We will indicate the changes in the proof of Theorem 3.9 which are necessary to prove the converse. Suppose that $E_{c}$ is not thin at infinity. It may be that $h$ and $I$ are not regulated, so we must proceed in another way. Let $e_{1}, \ldots, e_{n}$ be an orthonormal basis for $H$. For each $x$ in $G$ there must be an $i$ such that $\left\|h(x) e_{i}\right\| \geqq\|h(x)\| / n$. It follows from this that if $E_{i}$ is the set of all $x$ such that $\left\|h\left(x^{-1}\right) e_{i}\right\| \geqq c / n$, then $E_{c} \subset \bigcup_{i=1}^{n} E_{i}$.

If all $E_{i}$ are thin at infinity, $E_{c}$ would also be thin at infinity which is a contradiction. Thus for some $i, E_{i}$ is not thin at infinity. Now choose a sequence $V x_{n}$ as in the proof of Theorem 3.9 only substitute $E_{i}$ for $E_{c}$. Then leave $f$ unchanged, define $g_{n}(x)=e_{i} g\left(x^{-1} x_{n}^{-1}\right) \Delta\left(x_{n} x\right)^{-1 / 2}$ and proceed as before.

4. Twisted group algebras and induced representations. In [4] two of the authors introduced and studied a general type of twisted group algebra. We review briefly some of those results.

Let $A$ be a separable Banach *-algebra, $G$ a second countable locally compact group, $M(A)$ the double centralizer algebra of $A$, and $\operatorname{Aut}^{1}(A)$ the *-automorphisms of $A$ which together with their inverses have norm one. Let $T$ be a Borel measurable mapping from $G$ to $\operatorname{Aut}^{1}(A)$ and $\gamma$ a Borel map from $G \times G$ to $U(M(A))$ (the unitaries in $M(A))$ such that

(1) $(T(x) \gamma(y, z)) \gamma(x, y z)=\gamma(x, y) \gamma(x y, z)$,

(2) $(T(x) T(y) a) \gamma(x, y)=\gamma(x, y)(T(x y) a)$,

(3) $\gamma(e, x)=\gamma(y, e)=I, T(e)=I$,

for all $x, y, z$ in $G, a$ in $A$ ( $e$ the identity in $G$ ). The pair $(T, \gamma)$ is called a twisting pair for $(A, G)$ if the continuity condition of Proposition 2.1a of [4] holds.

We then form a new Banach *-algebra $L^{1}(A, G ; T, \gamma)$ by providing the Bochner integrable $A$-valued functions with the following product and involution:

$$
\begin{aligned}
(f \cdot h)(x) & =\int_{G} f(y)\left(T(y) h\left(y^{-1} x\right)\right) \gamma\left(y, y^{-1} x\right) d m(y), \\
f^{*}(x) & =\gamma\left(x, x^{-1}\right)^{*}\left(T(x) f\left(x^{-1}\right)\right)^{*} \Delta\left(x^{-1}\right),
\end{aligned}
$$

where, as before, $m$ is a left Haar measure of $G$ and $\Delta$ is the corresponding modular function. 
A number of special cases of this algebra have appeared in the literature. We will just mention that the group algebra of any second countable group extension of a group $G$ by a group $H$ can be written in the above form for $A=L^{1}(H)$.

We say that two pairs $(T, \gamma),\left(T^{1}, \gamma^{1}\right)$ as described above are equivalent if there is a Borel function $p$ from $G$ to $U(M(A))$ such that

(1) $p(e)=I$,

(2) $T^{1}(x) a=p(x)(T(x) a) p(x)^{*}, a$ in $A$,

(3) $\gamma^{1}(x, y)=p(x)(T(x) p(y)) \gamma(x, y) p(x y)^{*}$.

It can be shown that the algebra $L^{1}(A, G ; T, \gamma)$ depends, up to isometric *-isomorphism, only on the equivalence class of $(T, \gamma)$.

Now let $s$ be a nondegenerate *-representation of $A$ on a Hilbert space $H$. The induced representation $S$ of $L^{1}(A, G ; T, \gamma)$ on $L^{2}(G, m, H)$ is given by

$$
[S(f) g](x)=\int_{G} s[T(x) f(y)] s(\gamma(x, y)) g(x y) \Delta(y)^{1 / 2} d m(y) .
$$

The class of this representation, of course, depends only on the class of $(T, \gamma)$. In fact if $\left(T^{1}, \gamma^{1}\right)$ is related through $p$ to $(T, \gamma)$ as described above, let $M: L^{1}(A, G ; T, \gamma)$ $\rightarrow L^{1}\left(A, G ; T^{1}, \gamma^{1}\right)$ be defined by $(M f)(x)=f(x) p(x)^{*}$. One can easily prove that $M$ is an isometric *-isomorphism. Now let $S$ and $S^{1}$ be the representations of $L^{1}(A, G ; T, \gamma)$ and $L^{1}\left(A, G ; T^{1}, \gamma^{1}\right)$ respectively, induced on $L^{2}(G, m, H)$ by $s$. Finally, define a unitary $W$ on $L^{2}(G, m, H)$ by $(W h)(x)=s(p(x)) h(x)$. It is not hard to show that $W S W^{*}=S^{1} M$ and so, in this sense, the equivalence class of $S$ depends only on that of $(T, \gamma)$. In the group extension example, this definition coincides with Mackey's definition of induced representation. For the above results and other information see [4].

We will make the additional assumption that $T$ is strongly continuous at $e$ and $\gamma$ is jointly strongly continuous at $(e, e)$. This will not always be true, but in many cases there will be a pair $\left(T^{1}, \gamma^{1}\right)$ equivalent to $(T, \gamma)$ for which it holds. In particular, this follows for the group extension example from a theorem of Nagao [12] to the effect that one can always find a Borel measurable cross-section $t$ from the quotient group of a locally compact second countable group such that $t$ is continuous at $\bar{e}$ (quotient group identity) [14, Theorem 3].

THEOREM 4.1. Let $s$ be a nondegenerate *-representation of $A$ on $H$. Let $h_{a} \in L^{\infty}(G, m, B(H))$ be defined for each $a$ in $A$ by $h_{a}(x)=s(T(x) a)$. As usual, define $h_{a}^{\tilde{a}}(x)=h_{a}\left(x^{-1}\right)$. Then the following statements are equivalent:

(1) $S$ is C.C.R. $(S(f)$ is always compact).

(2) $s$ is C.C.R. and each $h_{a}^{\tilde{a}}$ almost vanishes at infinity.

Proof. (2) $\Rightarrow$ (1). Elements of the form $a \otimes f$ where $a$ is in $A, f$ is in $L^{1}(G)$ and $a \otimes f(x)=a f(x)$ are a total set in $L^{1}(A, G ; T, \gamma)$ and so it is sufficient to show that 
each $S(a \otimes f)$ is a compact operator. But

$$
[S(a \otimes f) g](x)=\int_{G} h_{a}(x) f(y) s(\gamma(x, y)) g(x y) \Delta(y)^{1 / 2} d m(y)=\left[Q_{k} K_{B f} g\right](x)
$$

where $k=h_{a}$ and $B=s(\gamma)$. Theorem 3.5 then shows $S(a \otimes f)$ is compact.

(1) $\Rightarrow(2)$. We first let $s$ be C.C.R. Suppose for some $a$ in $A, h_{a}^{\tilde{a}}$ does not almost vanish at infinity. Let $E_{c}$ be not thin at infinity, where $E_{c}$ is the set of all $x$ in $G$ such that $\left\|h_{a}(x)\right\| \geqq c$, and choose a symmetric neighborhood $V$ of $e$ such that if $x, y$ are in $V,\|T(x) a-a\|<c / 16$ and $\|a \gamma(x, y)-a\|<c / 16$. Choose an F.E. sequence $\left(V x_{n}\right)$ for $E_{c}$. Then the sets $x_{n}^{-1} V^{2}$ are disjoint for distinct $n$ 's. Define

$$
p: G \rightarrow U(M(A))
$$

by

$$
\begin{aligned}
p(x) & =\gamma\left(x_{n}^{-1}, z\right) & & \text { if } x=x_{n}^{-1} z \text { is in } x_{n}^{-1} V^{2} ; \\
& =I & & \text { if } x \text { is not in } \bigcup_{n} x_{n}^{-1} V^{2} .
\end{aligned}
$$

Form the equivalent pair $\left(T^{1}, \gamma^{1}\right)$ from $(T, \gamma)$ using $p$. Define $h_{a}^{1}$ by $h_{a}^{1}(x)$ $=s\left(T^{1}(x) a\right)$. If $S^{1}$ is the representation induced on $L^{1}\left(A, G ; T^{1}, \gamma^{1}\right)$ by $s, S^{1}(a \otimes f)$ for any $f$ in $L^{1}(G)$ is given by

$$
S^{1}(a \otimes f) g(x)=\int_{G}\left[h_{a}^{1}(x) f(y) s\left(\gamma^{1}(x, y)\right) g(x y) \Delta(y)^{1 / 2}\right] d m(y) .
$$

By previous remarks, to prove $S$ is not C.C.R. it is enough to show that $S^{1}(a \otimes f)$ is not compact for some $f$ in $L^{1}(G)$. Now $\left(h_{a}^{1}\right) \sim$ is greater than or equal to $c$ in norm on $E_{c}$, the same set as for $h_{a}^{\tilde{a}}$. We need only verify that $h_{a}^{1}$ and $B^{1}=s\left(\gamma^{1}\right)$ are regulated and we will be done, by Theorem 3.9. If $w_{1}, w_{2}$ are in $V x_{n}$ and $y_{1}, y_{2}$ are in $V$, then $w_{i}^{-1}$ is in $x_{n}^{-1} V$ and $w_{i}^{-1} y_{i}$ is in $x_{n}^{-1} V^{2}(i=1,2)$. Suppose $w_{i}=z_{i}^{-1} x_{n}$. Then $T^{1}\left(w_{i}^{-1}\right) a=T\left(x_{n}^{-1}\right) T\left(z_{i}\right) a$ and $\gamma^{1}\left(w_{i}^{-1}, y_{i}\right)=T\left(x_{n}^{-1}\right) \gamma\left(z_{i}, y_{i}\right)$. These relations follow from the definitions of $p$ and $\left(T^{1}, \gamma^{1}\right)$, and the relations satisfied by $T$ and $\gamma$. Thus

$$
\begin{aligned}
\| h_{a}^{1}\left(w_{1}^{-1}\right) B^{1}\left(w_{1}^{-1}, y_{1}\right)-h_{a}^{1}\left(w_{2}^{-1}\right) & B^{1}\left(w_{2}^{-1}, y_{2}\right) \| \\
& \leqq\left\|T\left(x_{n}^{-1}\right)\left(T\left(z_{i}\right) a \gamma\left(z_{1}, y_{1}\right)\right)-T\left(x_{n}^{-1}\right)\left(T\left(z_{2}\right) a \gamma\left(z_{2}, y_{2}\right)\right)\right\| \\
& \leqq \sum_{i}\left(\left\|T\left(z_{i}\right) a-a\right\|+\left\|a \gamma\left(z_{i}, y_{i}\right)-a\right\|\right)<c / 4 .
\end{aligned}
$$

It follows that $h_{a}^{1}$ and $B^{1}=s\left(\gamma^{1}\right)$ are regulated and so, by Theorem 3.9, $S^{1}(a \otimes f)$ is not compact for some $f$ in $L^{1}(G)$.

The only thing remaining to be proved is that if $S$ is C.C.R. then $s$ must also be C.C.R. Suppose that for some $a$ in $A, s(a)$ is not a compact operator. We may assume that $a$ is positive selfadjoint since if $s\left(a^{*} a\right)$ is compact, so is $s(a)$. Thus, $s(a)$ is a positive, selfadjoint, noncompact operator on $H$. We require the following:

Lemмa. If $B$ is a positive selfadjoint noncompact operator on a Hilbert space $H$, there is an orthonormal sequence $\left\{x_{n}\right\}, 1 \leqq n<\infty$, in $H$ such that $\inf _{n, m}\left\|B x_{n}-B x_{m}\right\|>0$. 
Proof. By spectral theory we can write $B=\int_{0}^{\infty} t d E_{t}$ for some projection valued measure $E$. There must be a $c>0$ such that the projection $E_{c}=\int_{c}^{\infty} d E_{t}$ is infinite dimensional, otherwise $B$ would be compact. Choose such a $c$ and let $\left\{x_{n}\right\}, 1 \leqq n<\infty$, be an orthonormal basis for the invariant subspace $E_{c}$. Then

$$
\begin{aligned}
\left\|B x_{n}-B x_{m}\right\|^{2} & =\left(B^{2}\left(x_{n}-x_{m}\right) \mid\left(x_{n}-x_{m}\right)\right) \\
& =\int_{c}^{\infty} t^{2} d\left\|E_{t}\left(x_{n}-x_{m}\right)\right\|^{2} \geqq c^{2} \int_{c}^{\infty} d\left\|E_{t}\left(x_{n}-x_{m}\right)\right\|^{2} \\
& =c^{2}\left\|E_{c}\left(x_{n}-x_{m}\right)\right\|^{2}=c^{2}\left\|x_{n}-x_{m}\right\|^{2} .
\end{aligned}
$$

Thus $\left\|B x_{n}-B x_{m}\right\| \geqq c\left\|x_{n}-x_{m}\right\|^{2}=c \sqrt{ } 2>0$.

Returning now to the theorem, we choose an orthonormal sequence $\left\{x_{n}\right\}$, $1 \leqq n<\infty$, in $H$ such that $\left\|s(a) x_{n}-s(a) x_{m}\right\| \geqq d>0$ for all $n, m$. Choose a compact symmetric neighborhood $V$ of $e$ in $G$ such that if $x, y$ are in $V, \| h_{a}(x) B(x, y) \Delta(y)^{1 / 2}$ $-s(a) \|<d / 3$. Again, let $g$ be the characteristic function of $V^{2}$, let $f=m(V)^{-1} C_{V}$, $g_{n}=x_{n} g$, and $w_{n}(x)=C_{V}(x) m(V)^{-1} \int_{V} h_{a}(x) B(x, y) x_{n} \Delta(y)^{1 / 2} d m(y)$.

By methods similar to those employed previously one can show (since $B(e, y)$ $=s[\gamma(e, y)]=I)$ that $\left\|w_{n}(x)-s(a) x_{n}\right\|<d / 3$ for all $x$ in $V$. Then

$$
\begin{aligned}
\| Q_{h_{a}} K_{B f}\left(g_{n}-\right. & \left.g_{m}\right) \|_{2}^{2} \\
& =\int\left[\left\|\int h_{a}(x) B(x, y)\left(x_{n}-x_{m}\right) f(y) g(x y) \Delta(y)^{1 / 2} d m(y)\right\|\right]^{2} d m(x) \\
& \geqq \int_{V}\left\|w_{n}(x)-w_{m}(x)\right\|^{2} d m(x) \\
& \geqq \int_{V}\left[\left\|s(a) x_{n}-s(a) x_{m}\right\|-\frac{2 d}{3}\right]^{2} d m(x) \\
& >d^{2} m(V) / 9>0 .
\end{aligned}
$$

This shows that the bounded sequence $g_{n}$ goes into a sequence with no converging subsequences under $S(a \otimes f)$. Thus this operator is not compact and the proof is complete.

We now give an alternate description of the main result for the case where the representation $s$ is irreducible. It is shown in [4] that for purposes of computing representations we may always assume that $A$ is a $C^{*}$-algebra. Thus we let $(T, \gamma)$ be a twisting pair for $(A, G)$ where $A$ is a $C^{*}$-algebra. Let $A^{\wedge}$ be the dual space of $A$, that is the space of equivalence classes of irreducible *-representations of $A$ with the hull-kernel topology (see [7]). It follows from Lemma 4.6 in [4] that $G$ acts on $A^{\wedge}$ through $T$ as a topological transformation group. If $s$ is a fixed irreducible representation of $A$ (with equivalence class also denoted by $s$ ), then there is a natural map $\pi$ from $G$ into $A^{\wedge}$ given by $\pi(x)=T(x) s$ where $(T(x) s)(a)=s\left(T\left(x^{-1}\right) a\right)$. We will say that $\pi$ is almost proper if for every compact set $\mathscr{K}$ in $A^{\wedge}, \pi^{-1}(\mathscr{K})$ is thin at infinity in $G$. 
Corollary 4.2. The representation $S$ of $L^{1}(A, G ; T, \gamma)$ induced from $s$ is C.C.R. if and only if (1) $s$ is C.C.R., (2) $\pi$ is almost proper.

Proof. It can be shown (see [5, Chapter 3]) that the sets in $A^{\wedge}$ of the form $\mathscr{K}(a, c)=\{s:\|s(a)\| \geqq c\}, a$ in $A, c>0$, are all compact and their interiors form a base for the topology of $A^{\wedge}$. Now using notation from Theorem 4.1, we see that the set of $x$ in $G$ such that $\left\|h_{a}^{\tilde{a}}(x)\right\| \geqq c, a$ in $A$, is nothing else but $\pi^{-1}(\mathscr{K}(a, c))$. Thus, each $h_{a}^{\tilde{a}}$ almost vanishes at infinity if and only if each $\pi^{-1}(\mathscr{K}(a, c))$ is thin at infinity. On the other hand, if this condition holds and $\mathscr{K}$ is an arbitrary compact set in $A^{\wedge}$, then for some $a_{1}, \ldots, a_{n}$ in $A$ and $c_{1}>0, \ldots, c_{n}>0$, we have

$$
\pi^{-1}(\mathscr{K}) \subset \bigcup_{i} \pi^{-1}\left(\mathscr{K}\left(a_{i}, c_{i}\right)\right)
$$

It is easy to show that finite unions and subsets of thin-at-infinity sets are thin at infinity, and so $\pi^{-1}(\mathscr{K})$ is thin at infinity. This completes the proof.

COROLlary 4.3. If $G$ is a countable discrete group and $s$ is in $A^{\wedge}, s$ induces a C.C.R. representation if and only if

(1) $s$ is C.C.R.

(2) Any sequence in $\pi(G)$ tends to infinity.

(3) The stability group $\{x \in G: \pi(x)=s\}$ is finite.

Proof. Clearly a set is thin at infinity in $G$ if and only if it is finite. Thus $\pi(G)$ intersects every compact set in only a finite set of points. The corollary then follows.

A glance at [4] shows that the above results specialize to interesting theorems on covariance representations of $C^{*}$-algebras and locally compact automorphism groups, as well as to algebras constructed from locally compact transformation groups. Perhaps the most interesting application, however, is to representations of group extensions. We elaborate on this application in the next section.

5. Induced representations of groups. We first interpret our main theorems in the context of induced representations of groups. Then we show that these can be strengthened and augmented if we add certain usual hypotheses. Let $e \rightarrow H \rightarrow G$ $\rightarrow K \rightarrow e$ be an exact sequence of second countable, locally compact groups. The action of $G$ on $H$ by inner automorphisms, $g \cdot h=g h g^{-1}$, induces action of $G$ on $L^{1}(H)$ and on $H^{\wedge}=L^{1}(H)^{\wedge}$ in the obvious ways. The following is a direct corollary of Theorem 4.1.

PROPOSITION 5.1. If $s$ is a representation of $H$ (unitary and strongly continuous on a Hilbert space), then the induced unitary representation $S$ of $G$ is C.C.R. if and only if

(1) $s$ is C.C.R.,

(2) for each $f$ in $L^{1}(H)$ the map

$$
g \rightarrow\left\|\int_{H} s\left(g^{-1} h g\right) f(h) d m(h)\right\|
$$

(which is constant on cosets of $H$ ) almost vanishes at infinity modulo $H$. 
We also have the following directly from Corollary 4.2.

Proposition 5.2. If $s$ is irreducible and $\pi(g)=g \cdot s$ maps $G$ into $H^{\wedge}$, then $S$ is C.C.R. if and only if

(1) $s$ is C.C.R.,

(2) $\pi^{-1}(\mathscr{K})$ is thin at infinity modulo $H$ for every compact set $\mathscr{K}$ in $H^{\wedge}$.

Now suppose that $H$ is type I and regularly embedded in $G\left(H^{\wedge} / G\right.$, the orbit space under the natural action of $G$ on $H^{\wedge}$, is a countably separated Borel space with the quotient Borel structure, see [11]). Fix $s$ in $H^{\wedge}$ and let $G_{s}$ be the stability subgroup of $s$ in $G$, i.e. all $g$ such that $g \cdot s=s$, and assume $G_{s}$ is normal. Then $H \subset G_{s} \subset G$ and it is proved in [2] that $G_{s}$ is closed. Let $t$ be an irreducible representation of $G_{s}$ whose restriction to $H$ is a multiple of $s$. We will always denote by $O(s)$ and $O(t)$ the orbits of $s$ and $t$ in $H^{\wedge}$ and $G_{s}^{\wedge}$ respectively induced by the action of $G$ on those normal subgroups. It is clear (cf. [2, p. 1112]) that the stability subgroup of $t$ in $G$ is just $G_{s}$. This means that the mapping $g \cdot s \rightarrow g \cdot t$ of $O(s)$ to $O(t)$ is well defined.

LEMMA 5.3. $O(s)$ and $O(t)$ are homeomorphic to each other and to $G / G_{s}$.

Proof. There is an obvious commutative diagram:

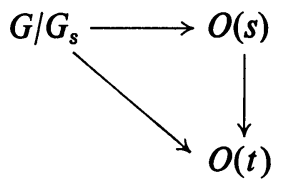

We know that all three maps are continuous since the two lateral ones are, all are bijective, and the map $G / G_{s} \rightarrow O(s)$ is a homeomorphism by [10, Theorem 1]. We complete the proof by showing that the map $O(s) \rightarrow O(t)$ is open. Let $Y$ be any subset of $G$, and suppose $x \cdot t$ is weakly contained in $\{y \cdot t: y$ in $Y\}$ ( $x$ an element of $G)$. Then $x \cdot t \mid H$ (restriction) is weakly contained in $\{(y \cdot t) \mid H: y$ in $Y\}([7$, p. 371]). For each $z$ in $G, z \cdot t \mid H$ is weakly equivalent to $z \cdot s$ (since $z \cdot t \mid H$ is a multiple of $z \cdot s)$, and so $x \cdot s$ is weakly contained in $\{y \cdot s: y$ in $Y\}$ and this shows that the map is open and completes the proof.

The following theorem shows that under the above hypotheses we can replace almost vanishing at infinity by vanishing at infinity and also find other equivalent conditions.

THEOREM 5.4. Let $s$ and $t$ be as above. For $f$ in $L^{1}\left(G_{s}\right)$ let $t(f)=\int_{G_{s}} f(x) t(x) d m(x)$. Then the following statements are equivalent:

(1) The induced representation $T$ of $G$ (induced from $t$ ) is C.C.R.

(2) $t$ is C.C.R. and for each $f$ in $L^{1}\left(G_{s}\right), y \rightarrow\|y \cdot t(f)\|$ vanishes at infinity modulo $G_{s}$.

3. $O(t)$ is closed in $\left(G_{s}\right)^{\wedge}$.

(4) The map $y G_{s} \rightarrow y \cdot t$ from $G / G_{s}$ to $\left(G_{s}\right)^{\wedge}$ is closed or proper (in the sense of Bourbaki, see [3]). (Note that we do not assume $O(s)$ is closed in $H^{\wedge}$.) 
Proof. (3) $\Leftrightarrow$ (4) follows from [3, Proposition 10.1.2].

(3) $\Rightarrow$ (2) is a consequence of Lemma 5.3 and Proposition 3.3.7 of [5].

(2) $\Rightarrow(1)$ is a corollary to Theorem 4.1 .

(1) $\Rightarrow$ (3). Again we resort to weak containment arguments. Let $r \in G_{s}^{\wedge}$ be weakly contained in $O(t)$. Then the induced representation $R$ is weakly contained in the set of representations induced from $\{x \cdot t: x \in G\}$ [8, Theorem 4.2] which is just $T$ (since all the representations induced from $x \cdot t$ are equivalent). Thus the support of $R$ (the closed set of all elements of $G^{\wedge}$ weakly contained in $R$ ) is precisely the set $\{T\}$ (closed, since $T$ is C.C.R.). Thus, $R$ is a multiple of $T$ and a theorem of Mackey [11, Theorem 8.1] can be used to show that $r$ is a multiple of $x \cdot t$ for some $x$ in $G$ and so $r=x \cdot t$ is in $O(t)$. This completes the proof.

REMARK 5.5. If the stability group $G_{s}$ is normal in $G$ for each $s$ in $H^{\wedge}$, then Theorem 5.4 gives necessary and sufficient conditions for the extension $G$ to be C.C.R., for under the hypotheses of this theorem each element of $G^{\wedge}$ is induced from some $t$ [11].

We now specialize to the case where $H$ is type I, regularly embedded in $G$ and $s$ in $H^{\wedge}$ is such that $O(s)$ is closed.

Let $M_{s}$ be the set of all $t$ in $G_{s}^{\wedge}$ such that the restriction of $t$ to $H$ is (unitarily equivalent to) a multiple of $s$, and let $M_{O(s)}=\bigcup_{x \in G} M_{x \cdot s \cdot}$.

Lemma 5.6. For any $s$ in $H^{\wedge}, M_{O(s)}$ is closed in $G_{s}^{\wedge}$ if $O(s)$ is closed.

Proof. Suppose $r$ in $G_{s}^{\wedge}$ is weakly contained in $M_{O(s)}$. As in Lemma 5.3 we can show that $r \mid H$ is weakly contained in $O(s)$ and since the latter is closed, supp $(r \mid H)$ $\subset O(s)$. One can use Theorems 7.6 and 6.3 of [11] (and the fact that $O(s)$ is a locally compact $T_{2}$ space on which $G_{s}$ acts trivially) to show that $\operatorname{supp}(r \mid H)$ is $\{x \cdot s\}$ for some $x$ in $G$, and thus $r \mid H$ is a multiple of $x \cdot s$. Hence $r \in M_{x \cdot s} \subset M_{O(s)}$.

TheOREM 5.7. Let $O(s)$ be closed in $H^{\wedge}$ and let $t$ be in $M_{O(s)}$. Then the following statements are equivalent:

(1) $O(t)$ is closed in $G_{s}^{\wedge}$.

(2) $t$ is C.C.R.

(3) $T$ is C.C.R.

Proof. (3) $\Leftrightarrow$ (1) and (3) $\Rightarrow$ (2) by Theorem 5.4.

(2) $\Rightarrow(1)$. Let $r$ in $G_{s}^{\wedge}$ be weakly contained in $O(t)$. As before $R$ is weakly contained in $T$. Lemma 5.6 says that we may assume $r$ is in $M_{O(s)}$, so $r \in M_{x \cdot s}$ for some $x$ in $G$. Clearly for some $z$ in $G$ we have $z \cdot t$ in $M_{x \cdot s}$. Fell has shown [9] that $M_{x \cdot s}$ is closed and it then follows from [9, Lemma 4] that $r$ is weakly contained in $z \cdot t$. Since $z \cdot t$ is C.C.R. (and thus $\{z \cdot t\}$ is closed), $r=z \cdot t$ and $r$ is in $O(t)$.

$(2) \Leftrightarrow(3)$ was proved differently by Fell in [9]. 
Our final result is a global form of Theorem 5.7. We review briefly the place of projective representations in the theory under discussion. For all results and definitions concerning projective representations we refer the reader to [11]. We remark first that the irreducible $\sigma$-projective representations of a group $G$ ( $\sigma$ a complex valued multiplier on $G$ ) are in one-one correspondence with certain of the ordinary representations of a group extension $G^{\sigma}$ of $G$ by the circle. The set of equivalence classes of all such irreducible $\sigma$ representations of $G,(G, \sigma)^{\wedge}$, is topologized by this correspondence. If $s$ is in $H^{\wedge}$, where $H \subset G$ is as before, then there is a multiplier $\sigma_{s}$ on $G_{s} / H$ such that

(1) $s$ extends to a $\left(p \sigma_{s}\right)^{-1}$-representation $s^{1}$ of $G_{s}\left(p: G_{s} \rightarrow G_{s} / H\right.$ is projection).

(2) The map $r \rightarrow s^{1} \otimes r p$ of $\left(G_{s} / H, \sigma_{s}\right)^{\wedge}$ to $M_{s}$ is a bijection.

It follows from [13] that the above mapping is continuous, so that if $t \in M_{s}$ and $t=s^{1} \otimes r p$, then $r$ will be C.C.R. if $t$ is C.C.R. It is proved in [1, pp. 176-177], that if $s$ and $r$ are C.C.R. then so is $t$.

Now suppose that $H$ is type I, regularly embedded in $G$, and the orbit space $H^{\wedge} / G$ (in the quotient topology) is $T_{1}$ (in particular $H$ is C.C.R.). Suppose also that for each $s$ in $H^{\wedge}, G_{s}$ is normal. Then applying Theorem 5.7 and the ideas of Remark 5.5, together with the above remarks we have

THEOREM 5.8. The following statements are equivalent:

(1) $G$ is C.C.R.

(2) For each $s$ in $H^{\wedge},\left(G_{s} / H, \sigma_{s}\right)^{\wedge}$ is C.C.R.

(3) For each $s$ in $H^{\wedge}$, each $t$ in $M_{s}$ is C.C.R.

\section{REFERENCES}

1. L. Auslander and C. C. Moore, Unitary representations of solvable Lie groups, Mem. Amer. Math. Soc. No. 62 (1966). MR 34 \#7723.

2. R. Blattner, Group extension representations and the structure space, Pacific J. Math. 15 (1965), 1101-1113. MR 32 \#5785.

3. N. Bourbaki, Livre III: Topologie générale, 4th ed., Chap. 1, Actualités Sci. Indust., no. 1142, Hermann, Paris, 1965. MR 39 \#6237.

4. R. C. Busby and H. A. Smith, Representations of twisted group algebras, Trans. Amer. Math. Soc. 149 (1970), 503-537.

5. J. Dixmier, Les $C^{*}$-algèbres et leurs représentations, Cahiers Scientifiques, fasc. 29, Gauthiers-Villars, Paris, 1964. MR 30 \#1404.

6. N. Dunford and J. T. Schwartz, Linear operators. I: General theory, Pure and Appl. Math., vol. 7, Interscience, New York and London, 1958. MR 22 \#8302.

7. J. M. G. Fell, The dual spaces of $C^{*}$-algebras, Trans. Amer. Math. Soc. 94 (1960), 365-403. MR 26 \#4201.

8. - Weak containment and induced representations of groups, Canad. J. Math. 14 (1962), 237-268. MR 27 \#242.

9. - A new proof that nilpotent groups are CCR, Proc. Amer. Math. Soc. 13 (1962), 93-99. MR 24 \#A3238.

10. J. Glimm, Locally compact transformation groups, Trans. Amer. Math. Soc. 101 (1961), 124-138. MR 25 \#146. 
11. G. W. Mackey, Unitary representations of group extensions. I, Acta Math. 99 (1958), 265-311. MR 20 \#4789.

12. H. Nagao, The extension of topological groups, Osaka Math. J. 1 (1949), 36-42. MR 11, 10.

13. I. Schochetman, Topology and the duals of certain locally compact groups, Trans. Amer. Math. Soc. 150 (1970), 477-489.

14. R. C. Busby, On the equivalence of twisted group algebras and Banach *-algebraic bundles (submitted).

Department of Mathematics, Drexel University, Philadelphia, Pennsylvania 19104

Department of Mathematics, Oakland University, Rochester, Michigan 48063

Executive Office of the President, Washington, D.C. 20504

Current address (Smith): Department of Mathematics, Oakland University, Rochester, Michigan 48063 